\title{
Exchanging Bodily Fluids: \\ Transubstantiations in Contemporary Pornography
}

\author{
Helen Hester
}

\section{Introduction: The Critical Response to Gagging in Pornography}

Gagging is arguably one of the most high-profile elements of the contemporary pornographic landscape, with the involuntary muscular spasms resulting from irrumatio (or rough deep throat fellatio) being showcased in even relatively mainstream gonzo porn releases. Titles such as Asa Akira is Insatiable 3 (2012) and Flesh Hunter 11 (2012), for example, feature irrumatio as part of a wider repertoire of gonzo sex acts, suggesting that whilst the stimulation of the gag reflex may not always in itself constitute a discrete pornographic "number" (Williams 72 ), ${ }_{1}^{1}$ it is at least a signature step in gonzo's established choreography. Gagging's profile is further enhanced by the fact that, in addition to operating as a key generic component, irrumatio has generated a popular pornographic subgenre of its own. The adult video-on-demand website Hot Movies, for example, lists "Gagging" as a distinct category of "Oral," with a total of 311 dedicated entries. ${ }^{2}$ A number of extensive series are devoted to the practice, including Chokers and Gaggers, Face Fucking Inc., and Throat Gaggers, the latter of which promises "sluts [...] covered in slime" and "Blowjobs as a full contact sport!"

Perhaps understandably given the at times deliberately inflammatory and provocative framing of these practices within the industry, much of the commentary upon gagging as a pornographic practice focuses upon its apparent misogyny. Critics concentrate upon the potentially troubling manner in which irrumatio stages gendered power relations (or more accurately, gendered power imbalances) within an overtly sexualized context. Robert Jensen, for example, presents "exclusively aggressive 'throat fucking'" as illustrative of pornography's pervasive hostility towards women (44), whilst Pamela Paul suggests that "multiple oral sex scenes in which the girl is shown to choke on genitalia and semen" are part of a worrying trend towards the acceptance of increasingly extreme and misogynistic content in hard core (8). 
The academic and activist Gail Dines, meanwhile, uses irrumatio as an example of one of contemporary adult entertainment's most damning excesses. She positions it at the heart of gonzo's generic formula - "There are $\mathrm{X}$ number of minutes given over to oral sex, often leading to the woman gagging, then anal, then double penetration, and then ejaculation" (Dines 68) - whilst linking this formula to the numbing of male empathy and the eroticizing of female subjugation. She also gives over several early pages to a discussion of Gag Factor (a specialist line of gagging porn), during the course of which she offers detailed and disturbing descriptions of the product. These descriptions are, it would seem, intended to enlighten her readers regarding the extremity of today's pornographic content whilst also rendering them particularly receptive to an antipornography message:

On the site are hundreds of pictures of young women with penises thrust deep into their throat. Some are gagging, others crying, and virtually all have faces, especially their eyes, covered in semen. The user is bombarded with images of mascara running, hair being pulled, throats in a vicelike grip, nostrils being pinched so the women can't breathe as the penis fills the mouth, and mouths that are distended by either hands pulling the lips apart or penises inserted sideways. (Dines xix-xx)

This site is then positioned as part of a wider pornographic culture - a culture in which "the man makes hate to the woman" and "each sex act is designed to deliver the maximum amount of degradation" (Dines xxiv-xxv).

In the work of commentators such as Dines, Jensen, and Paul, then, irrumatio has a clear and monosemic message, and the scenarios of gendered violence and male power that it apparently enacts are positioned as evidence of the aggressive tendencies of pornography in general. However, I would argue that there is more to gagging than "an intensification of the blowjob, a deep penetration of the mouth, an obvious staging of male power" (Stüttgen, "Disidentification in the Center of Power" 52); in fact, the manner in which this act is represented raises a number of interesting questions about the operation and limitations of the generic visual language of photorealistic hard core. In particular, the incorporation of corporeal paroxysms and the utilization of abject bodily fluids within representations of irrumatio would seem to prompt a re-examination of certain critical assumptions about the aesthetics of pornography. In what ways might the 
seemingly one-dimensional imagery of gagging serve to open up a discussion about the generic conventions of porn? How are abject substances coded, represented, and invested with significance, and how might they be said to contribute to pornography's attempt to visualize female desire? Drawing upon Linda Williams's scholarly work on pornography, along with examples from contemporary adult entertainment, this essay will attempt to provide at least a partial answer to these key questions.

\section{An Alternative Aesthetics of Pornographic Fluids}

Semen is widely accepted as the pornographic fluid par excellence. Not only does it have little cultural visibility beyond the confines of adult entertainment, ${ }^{3}$ but it plays a key role in the structuring of many contemporary porn scenes. Numerous Porn Studies scholars have identified the centrality of male ejaculate within the current conventions of the genre; Linda Williams, for example, discusses porn's "reliance on visible penile ejaculations (money shots) as proof of pleasure," describing it as "one of the most significant features of the form' (8), and remarks upon the manner in which the money shot has 'assumed the narrative function of signalling the climax of a genital event" (93). In a similar vein, Pasi Falk discusses the "anti-representational logic of hardcore pornography" (17), in which "scenes of explicit ejaculation" are exploited for their apparent indexical evidentiality (19), whilst Lisa Jean Moore and Juliana Weissbein suggest that the pornographic "male gaze is constantly reinforced through the ejaculating of a masculine glaze, a glaze that coats the other and the self with a glossy, slippery substance that modifies social relations" (78). However, whilst gagging pornography has by no means eschewed a reliance on the external cum shot - the signalling of male climax typically retains a privileged significance in terms of narrative sequencing - I would argue that the subgenre has developed its own aesthetics of fluids. In fact, it utilizes a visual language which (contingently, problematically, and temporarily) displaces the male cum shot, whilst also mirroring and extending it, as an alternative set of abject bodily fluids are pushed to the fore.

We might draw an example from the on-going series Throat Gaggers, a specialist line currently on its 14th instalment. The opening scene of Throat Gaggers 13 (2007) features porn star Allison Pierce alongside the male performer Johnny Fender and, from behind the camera, the director Juan Cuba. The scene begins with Pierce pulling up to a car park and talking briefly with the director, before heading inside to the men's bathroom. Here, she discovers Fender waiting for her in one of the 
stalls, and the action quickly proceeds to the subgenre's standard fare of multiple rough blowjobs and acts of irrumatio. During the course of the scene, we witness Fender dipping his penis in and out of his co-star's mouth, often wholly withdrawing it in order to elicit strands of saliva and mucous. He repeatedly rubs his cock around the exterior of Pierce's mouth and slaps it across her cheeks and chin, resulting in the visible smearing of her face with fluids. She gags noisily and sporadically throughout the performance and, at around the 10 minute mark, we hear her coughing and spluttering as the male performer performs irrumatio whilst holding her in place by her hair. The scene concludes with a facial cum shot.

This scene includes many of the standard generic markers of contemporary gagging porn; the emphasis is largely shifted from genital intercourse and close-up "meat shots" (Williams 72) of the penis entering the vagina or anus in favour of shots of the face and, particularly, of the deep penetration of the mouth. The inclusion of the images and sounds of choking and retching emphasizes the activation of the gag reflex, whilst the inclusion of dialogue from the male participants deliberately intensifies the viewer's sense of the eroticisation of force: the phrase "Choke on that cock," for example, is repeated like an erotic litany. One of the key market differentiators of gagging porn, however, is its focus upon an alternative set of bodily emissions: whilst the scene concludes with the generically mandated money shot, the fluids which are consistently privileged throughout are those produced by the female performer. In the course of the rough and repeated acts of irrumatio, she produces saliva, mucous from the nose and throat, and tears. The camera does not attempt to ignore these corporeal by-products, but lingers upon them, and adjusts its position in order to better represent them. The director dwells upon Pierce's face throughout, but comes in particularly close when the first tear of exertion rolls down her cheek. In the case of Throat Gaggers 13, the female's fluids are the real star; her made-up face becomes moist with its own secretions, as her male co-star accompanies her performance with repeated verbal cues: "I love that fucking spit," "Look at that spit, look at that spit, look at that spit."

This interest in representing an alternative set of bodily substances - a number of which meet Julia Kristeva's definition of the abject as "something to be ejected, or separated" $(127)^{4}-$ is not unique to Throat Gaggers 13. In fact, such an interest is fairly pervasive within gagging pornography, as evidenced by the manner in which many of its products are marketed. Many of the films and series within this subgenre share a similar visual logic in terms of their design and branding, for example, 
and this includes a focus upon capturing the various secretions of the female performers' faces. The stills used for DVD box covers, promotional web content, or other paratextual materials typically include details like spit bubbles, nasal secretions, or globules of mucous. The box cover for the 2010 movie Gag Factor 31 (displayed with others on the Gag Factor website) shows a heavily made up blonde woman with blood-shot eyes, situated over the body of her male co-star in something like the 69 position. She is looking into the camera, with her lips around the head of his erect penis, and a thick string of viscid mucous descends from her nose, running the length of the shaft of the penis and down onto the testicles. There is also a bead of cloudy white mucous (or is it ejaculate?) hanging from a strand of her hair.

We find evidence of a similar aesthetic not only throughout the other box covers in the Gag Factor series, but also in the marketing materials of other specialist lines. Choke on my Cock, Throated, and Black Gag, to name just three, all feature photographs of the faces of their female performers smeared with a profusion of semi-opaque bodily secretions. The visuals used in marketing these lines are reinforced by the advertising copy. That is to say, the linguistic rhetoric which tends to surround adult entertainment's representation of irrumatio includes a similar focus upon an alternative set of pornographic bodily substances. The copy used to promote Jonni Darkko's Sloppy Head 4 on the Hot Movies website, for example, promises scenes that are "just dripping with intense deep-throat gagging, phlegm, spit bubbles... and enough saliva to drown several cocks." The site's blurb for Choke On My Cock, meanwhile, states that "There's so much spit, more than enough to cover the cocks and balls and even compete with the ocean of cum that slides down their face and tongues." The fluids of the face, then, play a key role in selling depictions of irrumatio to the consumer. These emissions, it would appear, are at the heart of gagging porn's generic appeal, to the extent that they "even compete" with the more conventional representation of seminal fluids.

\section{Displacements and Transubstantiations: Linda Williams's "Frenzy of the Visible"}

How, then, are we to understand the prominence of this alternative set of bodily emissions within gagging pornography? Can it be related to the external ejaculation and to the generic convention of the money shot? In some ways, I would agree with Tim Stüttgen's comment that "Through more bodily fluids like spit and tears, an attempt is made to somehow double the effect of the cum shot" (Stüttgen "Before Orgasm," 12): the focus upon tears, saliva, and other forms of facial slime works 
to replicate and amplify the representation of male ejaculate reflected in the visual comparisons it invites. The emissions drawn out by vigorous deep throat fellatio redouble the conventional money shot to some extent; they target the face, smearing it with the viscous and abject by-products of sexual contact. The pleasures of the money shot - supposedly the pleasures of "marking territory and claiming ownership" (Moore and Weissbein 78) - are therefore extended. The money shot is no longer contained within the final act of a pornographic performance, but is instead laced through the scene as a whole, gradually intensifying as the oral penetrations, and concomitant choking and gagging, build throughout the course of the pornographic performance.

However, there is more to explore here, for whilst the visual depiction of facial emissions can in some ways be seen to prolong the effect and visual impact of the external ejaculation, it also to some extent substitutes for or displaces it. As the money shot is extended, and its generic and aesthetic function performed by an alternative variety of fluids, semen loses its uniquely privileged role in signalling the woman as "a site to be marked (leaving the ejaculator unmarked)" (Moore and Weissbein 79). The position of semen, in other words, is usurped by bodily secretions produced by the woman herself. This may prompt us to question why gagging pornography requires a supplement to the facial cum shot at all. In what ways does the representation of external penile ejaculation fail to meet the demands of the subgenre? How does gagging porn's use of facial slime work to augment the pleasures of pornography more generally? We can find at least a partial answer to these questions within Linda Williams's ground breaking study Hard Core: Power, Pleasure, and the "Frenzy of the Visible".

In this text, Williams traces the evolution of the cum shot back to the earliest days of photography, detecting its origins within a "cinematic will-to-knowledge" that has been operational "ever since photographer Edward Muybridge first threw the image of naked moving bodies on the screen of his lecture hall and ever since Thomas Edison ordered his technicians to photograph a sneeze" (101). Photography as a form, she argues, has long been driven to seek "visual evidence of the mechanical "truth" of bodily pleasure caught in an involuntary spasm" (101), and, in the case of hard-core pornography, this is extended to "the ultimate and uncontrollable - ultimate because uncontrollable - confession of sexual pleasure in the climax of the orgasm" (101, original emphasis). The appeal of the money shot, then, can be at least partially attributed to the pleasures 
of witnessing the "frenzy of the visible" (Williams 7) - seeing the authentic convulsions of the human body in a state of intensity graphically depicted in photorealistic detail.

This is complicated somewhat when the issue of gender is brought to bear, for whilst the representation of external ejaculation no doubt facilitates a visual encounter with the male sexual climax, the "truth" of female pleasure remains un-visualized. This is particularly troubling for heterosexual pornography, where the genre is largely positioned as being about encountering the female body and its pleasures. With the money shot, as Williams puts it, adult entertainment's "visibility extends only to a knowledge of the hydraulics of male ejaculation, which, though certainly of interest, is a poor substitute for the knowledge of female wonders that the genre as a whole still seeks" (Williams 94). But if heterosexual photorealistic pornography is primarily invested in exploring (and exposing) the secrets of the female body, then why has the depiction of semen in particular become so crucial to the genre's visual vocabulary of pleasure ${ }^{5}$

For Williams, this question concerns the limitations of pornography as a moving image genre and, indeed, the limitations of cinema as a medium. Despite its seeming explicitness, adult entertainment draws our attention to the unavoidable constraints associated with the practice of making visible. As Karen Boyle puts it, "Pornography is produced according to a principle of maximum visibility, not maximum sensation: the goal is to make sure the viewer gets a good look" (206). In necessarily privileging the ocular, filmic hard core struggles to generate a successful and convincing visual vocabulary of female desire. Whilst, as Falk remarks, the "phallic system" can demonstrate the "actual presence of (sufficient) sexual arousal" (18), and can therefore function as "an 'indexical sign' in [the] Peircean sense" (19), a female porn performer's pleasure is less easy to verify within the conventions and constraints of the medium. It cannot present or deliver itself unequivocally to the lens. After all, "the female partner is able to lie or act" (Falk 18), thus rendering the task of generating "a convincing representation of female sexual pleasure in the absence of evidential signs" particularly problematic (Falk 19).

This inability to capture female desire, pleasure, and climax using porn's conventional representational strategies results, somewhat counter-intuitively, in the genre's celebration of and (over-)reliance upon the spectacle of seminal ejaculation. That is to say, the genre compensates for its failure to demonstrate and prove certain kinds of orgasmic pleasures with an overemphasis 
upon others. It enacts the fetishistic exchange of the male climax for the female; it is, to quote Williams, a "form of cinematic perversion" and a "fetish substitute for less visible [...] instances of genital connection" (95). In short, then, adult entertainment represents "the obsessive attempt of a phallic visual economy to represent and "fix" the exact moment of the sexual act's involuntary convulsion of pleasure" (Williams 113), and it displaces its interest in the supposedly invisible female orgasm onto the fetishized representation of external ejaculation.

In the case of pornographic depictions of irrumatio, then, we are faced with a further complication of what is already a fairly complex contortion of pornography's underlying investments. As we have seen, gagging pornography uses the secretions of the throat, mouth, eyes, and nose to both extend and prefigure the conventional cum shot, smearing the faces of female performers with their own emissions. The pornographic principle of maximum visibility is therefore temporarily redirected, as an obsession with the emissions of the male body at the moment of orgasm is supplanted by an obsession with abject facial slime. What we have here is a double displacement, from the obscure moistenings of the female orgasm onto the more visually legible fluids of penile ejaculation, and then from this male cum shot onto the sexually-derived secretions of the face. In other words, we go from female genital fluids to female facial fluids, via male ejaculatory fluids.

This is strongly suggested by certain moments in Allison Pierce's scene in Throat Gaggers 13. At one point, her co-star instructs her to "rub that spit in your pussy;" Pierce scoops up the viscid slime from his cock with her fingers and does as requested, repeatedly touching her vagina and withdrawing her hand to show that cloudy white strands of mucous have been (or are being) transferred from her hand to her labia. The secretions of the woman's face and mouth are first transferred to the penis, where they function as something like a non-climatic money shot, before they are conveyed to the vagina. Here, bodily fluids are literally transferred, as wetness is returned to the female genital organs in a more visually accessible form. Interestingly, this effect of displacement is also enhanced via the scene's comparable use of sound. There is a cut nearing the conclusion of the scene, during which the camera jumps from a pseudo-POV shot of Pierce on her knees, taken from just behind the male performer's body, to a close-up of her masturbating with her hand inside her purple knickers. This image is accompanied by a soundtrack of churning wetness, which seemingly coincides with the rhythmical movements of her hands. For the duration of the shot (a mere two seconds or so), it appears as if the sounds are emanating from her vagina - 
a soundtrack of moist arousal and authentic female pleasure - before the camera pans up to show their true origin; Fender, gripping her hair and performing irrumatio. The sounds of the sloppy deep throat blow job momentarily overlay the image of Pierce touching herself, allowing for a temporary sonic displacement of oral moisture onto the vagina, and briefly facilitating a fantasy of accessing and documenting the pleasures of the female body.

It is also worth noting, I think, that this displacement of bodily secretions is mirrored by shifts in the representation of involuntary corporeal convulsions. The choking cough and the dry-heave play a significant role within irrumatio-centric adult entertainment. Many of its sexual technologies appear to be angling for these kinds of involuntary response, from the depth, speed, and duration of the male performer's thrusts to the trend for "nostrils being pinched so the women can't breathe" (Dines xx). For example, in the opening scene of 2004's Gag Factor 15 (and, indeed, throughout the various instalments of this long running series), we see the camera repeatedly zoom in on porn performer Ashley Blue as she retches and coughs, retaining a tightly cropped close-up of her face whenever she appears to experience an involuntary bodily paroxysm of any kind.

This is, at least in the examples I have mentioned here, a common trait of gagging pornography, and reiterates the logic of the two-fold exchange of bodily fluids in operation within the subgenre. In other words, I would contend that this type of adult entertainment can again be seen to plot a circuitous path back to the female body via the pornographic frenzy of the visible. The ecstatic shudders of the female body beside itself become the convulsions of a visually verifiable penile ejaculation, and these are in turn displaced by the similarly involuntary and telegenic muscular spasms of the agitated gag reflex. In this sense, the aesthetics of contemporary gagging pornography enacts not only an exchange of bodily fluids, but an exchange of corporeal paroxysms.

\section{Provoking Reactions and Moving the Body}

This correlation of a pornographic interest in female sexual response with an interest in the sexually explicit depiction of women choking, gagging, and crying requires further unpacking. Could the representation of two things as diverse as an orgasm and a dry heave really be said to tap into a common set of scopo- or epistemophilic spectatorial investments? It would be worth reflecting upon the idea of displacement in greater detail here, I think, for whilst the connection of two 
seemingly such disparate kinds of bodily response may strike one as counter-intuitive, a number of theorists of pornography have suggested that a set of exchanges, replacements, and substitutions may be at work within the content and reception of the genre. Williams, for example, detects a certain slippage of adult entertainment's generic interests within one of its highest profile (and most controversial) categories - sadomasochistic pornography.

Discussing some of the various forms, types of content, and representational strategies deployed within the S/M subgenre, Williams refers to the "displacement of a hard-to-see pleasure onto an easier-to-see, and apparently similarly involuntary, response to pain" (203). As with gagging porn's transference of the orgasmic shudder onto the paroxysmic retch or dry heave, certain kinds of S/M imagery can be seen to enact a comparable displacement. That is to say, they relocate porn's "obsessive attempt [...] to represent and "fix" the exact moment of the sexual act's involuntary convulsion" (Williams 113) from one instinctive corporeal response to another. This extension of the frenzy of the visible may prompt us to reflect upon how we currently characterize pornography's content and generic pleasures. Conventionally, as Lawrence O'Toole insists, people assume that contemporary "porn is a sex thing" (342), but the very possibility of this kind of displacement would seem to suggest the potential for a more diverse set of investments. In other words, the affective kick that comes with witnessing the involuntary reactions and convulsions of the human body is not connected exclusively to the activities of genital sexuality, but may segue into other varieties of bodily reflex.

In a move that is particularly useful for our purposes, Williams extends her argument about S/M porn's portrayal of involuntary corporeal paroxysms to the subgenre's use (or potential use) of bodily fluids. In a discussion that encompasses horror, exploitation cinema, and extreme or violent porn, she touches upon the manner in which apparently diverse discourses of authenticity and bodily event can be related back to the pornographic frenzy of the visible. When read in the generic context of adult entertainment as opposed to that of horror, she argues, "a flinch, a convulsion, a welt, even the flow of blood itself, would seem to offer incontrovertible proof that a woman's body, so resistant to the involuntary show of pleasure, has been touched, "moved" by some force" (194). ${ }^{6}$ In this theoretical re-imagining, blood replaces semen as the source of pornography's authenticity effect; whilst the money shot proves (according to the particular 
standards and conventions of the genre) that pleasure really has been achieved and experienced, it is posited that blood can also be used to achieve the same effect.

This obviously intersects with our analysis of the use of facial fluids within pornographic depictions of irrumatio. By dwelling upon the generation and profusion of these abject excretions - by presenting the very moment of their emergence in graphic detail and loving close-up - gagging porn provides its viewers with a kind of proof that the woman's body has really experienced something. The deliberate foregrounding of snotty noses, saliva-smeared mouths, and watering eyes is nothing if not an attempt to show that the female performer has been moved by some irresistible sexual force. As Williams states,

Hard core desires assurance that it is witnessing not the voluntary performance of feminine pleasure, but its involuntary confession. The woman's ability to fake the orgasm that the man can never fake (at least according to certain standards of evidence) seems to be at the root of all the genre's attempts to solicit what it can never be sure of: the out-of-control confession of pleasure, a hard-core "frenzy of the visible". (50)

In the case of gagging porn, the female body's involuntary confession acquires a visual language of its own. Via the exchange of orgasm and vaginal wetness for retching and facial discharges, the authentic experiences of the female body are rendered cinematically legible. Indeed, not only do the fluids triggered and extracted by irrumatio possess the requisite spectacular quality demanded by the moving image form but, crucially, they also read as (sufficiently) convincing within porn's ocular economy.

We have established, then, that the generic function of the cum shot can be usurped by alternative authenticating fluids, be these blood or saliva. By appearing convincing, and indeed by simply appearing (that is, by yielding to capture by the lens), these fluids provide gagging porn with an appropriate representational strategy for addressing one of contemporary adult entertainment's biggest generic challenges. Where facial secretions arguably have the advantage over a substance like blood, however, is that they can be easily inserted into porn's existing aesthetic repertoire and can operate within the adult industry's mainstream. The flow of real blood from real injuries should 
by no means be considered a significant part of the contemporary mainstream pornographic landscape; in my experience, such fluids have extremely limited visibility outside of non-niche adult entertainment, and even certain dedicated S/M sites will avoid things like blood and needle-play on the grounds that they are "distasteful or dangerous" (Mooallem 6). ${ }^{7}$

The kind of specialist S/M content that does depict "the flow of blood itself" (Williams 194), meanwhile, is often markedly distinct from gonzo in terms of audience, aesthetics, and content; indeed, we could argue that it is at the other end of the pornographic spectrum, displaying a fundamentally divergent understanding of what counts as sexual activity. In his essay "'Choke on it Bitch!': Porn Studies, Extreme Gonzo and the Mainstreaming of Hardcore," Stephen Maddison identifies a tendency within contemporary gonzo to ignore the traditional "progression from arousal and foreplay, through to penetration and orgasm" in favour of "mechanized cycles of penetration" that are organized "solely according to the logic of penile stimulation, by hand, mouth, vagina or anus, and multiple combinations thereof" (49). Gonzo pornography, Maddison argues, is preoccupied by the act of penetration to the exclusion of all other forms of sexual behaviour.

S/M pornographies, meanwhile, are less concerned with this kind of sexual activity, often eschewing scenes of penetration altogether. R. D. K. Herman argues that

Although by definition BDSM has sexual connotations, it is important to note that BDSM practices are not necessarily directly sexual and BDSM "scenes" may involve little or nothing that would be defined as sexual activity (genital stimulation). Instead, the defining characteristic is the power exchange whereby one person willingly relinquishes some degree of control over his or her body to another person. (92)

Linda Williams agrees, stating that BDSM-themed porn "often displaces the display of genital sex onto elaborate scenarios of punishment" (303). The representation of blood-play within S/M pornography, then, is quite separate from the gonzo material that today makes up adult entertainment's mainstream; ${ }^{8}$ so much so, in fact, that it typically displaces not only convulsions 
and fluids, but also genital sex itself. Whilst blood may have the capacity to operate as an authenticating fluid, it can do so only within very limited contexts and for very niche audiences.

Whereas the conventions surrounding the depiction of blood may be somewhat at odds with the norms of contemporary porno, however, the representation of facial fluids fits into a more accepted and widely disseminated set of practices. These fluids are presented as the direct result of oral sex - the blow job, of course, has enjoyed a particularly high degree of generic visibility since the release of Deep Throat in 1972 - and stem from the penetration of an existing orifice rather than relying upon the creation of new bodily openings via the cutting of skin. This means that the pornographic portrayal of irrumatio can exploit the transgressive frisson attached to the gendered dynamics of force, pleasure, and power, whilst at the same time retaining a useful distance from the kinds of violence that the wider culture is quickest to condemn as criminal or pathological.

The emissions of the face also have the advantage of sharing certain visual qualities with semen. As we have seen, the secretions of the nose and throat often display a similar viscosity and opacity to male ejaculatory fluid, whilst their colouration and placement (around the mouth and over the face) roughly approximates the aesthetics of the cum shot. Indeed, these two sets of abject fluids penile and facial - can at times be seen to merge as part of a pornographic focus upon wetness, both within specialist gagging films and within those more general works of gonzo hard core which incorporate irrumatio. Rocco Siffredi's Rocco: Animal Trainer 8, for example, uses rough deep throating and gagging as part of its wider generic repertoire, and provides a particularly clear example of how moistness might be displaced from the interior of the vagina to the more visually accessible location of the skin. In scene 3, for example, sweat and copious amounts of baby oil combine with the by-products of irrumatio to create a general aesthetics of wetness that is not limited to the cum shot; specks of moisture even appear on the camera lens at some points as Siffredi and his co-star Laura Orsoia perform.

What is crucial here is that the use of facial fluids offers visually decipherable evidence that the female bodily interior really has been moved and affected by a sexual act, but unlike blood, this evidence operates within the familiar and generically readable aesthetic perimeters of the money shot. This, of course, has its own limitations and raises its own questions; in attempting to visualize the stimulation undergone by the female performer's body according to those conventions 
typically applied to external penile ejaculation, the "truth" of feminine sexual experience is reduced to a facsimile of male pleasure. This is something that the film scholar Tanya Krzywinska picks up on in her essay "The Dynamics of Squirting: Female Ejaculation and Lactation in Hardcore Film." Discussing the lactation porn film Erupting Volcanoes, she notes that

Within hardcore the male come-shot is an undeniable sign of authentic sexual pleasure and here lactation is subject to the same paradigm (this is of course a male genital paradigm). The imposition of this framework on the lactating woman tempers the radical and powerful otherness of the mother's body and the mother's desire again disappears off-scene. (36)

With gagging as with lactation, the sensations of the female body are contained within adult entertainment's existing generic framework, resulting in little more than the mirroring of male sexual mechanics. ${ }^{9}$ Whilst the pornographic use of facial fluids as an extension of, or stand-in for, the cum shot may be traced back to an interest in the authentic sexual experiences of the female body, then, it ultimately fails to make any advance in its attempts to screen female affect.

\section{Conclusion: Future Directions for Theories of Gagging Porn}

In this article, I have argued that the fluids elicited by irrumatio and focussed upon within gagging pornographies operate as a prefiguration, extension, and displacement of the conventional cum shot, which is itself a fetishistic substitute for a female orgasm which remains perniciously resistant to photorealistic forms of representation. The abject secretions emitted from the faces of female performers are, I have argued, subject to a double displacement, as the attempt to engage with the experiences of the woman's body are diverted or re-routed through the existing generic conventions for representing pleasure in porn. My critical response differs from many popular approaches to the subgenre in that it positions the representation of gagging in pornography not so much as an unambiguous demonstration of male brute force, but as a relatively complex (not to mention unstable) strategy for externalizing and making visual the female performer's sensations and affects - sensations and affects which would otherwise be illegible according to the generic conventions of adult entertainment. 
It is entirely understandable that critics should interpret (and condemn) the representation of gagging and irrumatio within porn according to the manner in which it seems to use and abuse female performers, and propagate damaging ideas about the acceptability of the sexual mistreatment of women by men. Certainly, the acts depicted do not typically read as enjoyable for the women involved, and the manner in which gagging porn is marketed stresses a deliberate disinterest in (or hostility towards) catering to female pleasure. The marketing copy for the Italian gagging release Fuck Her Throat \#2 (n.d.), for example, states "There tears become lubrication for the next ram into their faces. Who gives a fuck if they can breathe... (sic)" (Hot Movies). This displays an overt disregard for women's comfort, let alone pleasure, and it is important that feminist critics pick up on and respond to the obvious revelling in misogyny that such texts represent.

Nonetheless, as this article has shown, gagging porn can be said to be (indirectly, circuitously) preoccupied by the challenges posed by capturing female pleasure and/as the frenzy of the visible. This is perhaps reflected by the following remark from Gail Dines, made in the context of her discussion about the lack of empathy and respect male performers demonstrate towards their female co-stars:

The only time men [in pornography] moan, grunt, or writhe is when they are about to ejaculate; the rest of the time they methodically thrust their penises into the woman's orifices with a look of deep concentration on their faces. This can get very bizarre, especially in an oral sex scene where a stoic man gags a woman by thrusting his penis deep in her mouth, yet she is the only one having orgasmic responses. ${ }^{10}$ (xxiv)

It is no coincidence, I think, that Dines should note that only gagging porn's female performers should exhibit the signs of sexual excitement, ${ }^{11}$ if my thesis regarding the displacements enacted by gagging porn is correct, then we must acknowledge that much of this subgenre is really about the body of the woman. The man - the figure that this type of pornography apparently seeks to celebrate and serve - is of secondary importance; it is her pseudo-orgasmic response, and her bodily fluids, which ultimately seem to be most compelling. 
This essay is indebted less to the anti-pornography polemics of people like Dines than it is to work currently being undertaken within cultural studies - particularly in the field of Porn Studies. There is already some excellent material seeking to address the challenges posed by attempting to visualize the fluids of female arousal, including essays by Tanya Krzywinska, Deborah Shamoon, and Eugenie Brinkema. However, there is still more to be done. My own article leaves many questions unanswered, particularly when it comes to the effect of sexual orientation upon the content and spectatorial pleasures of gagging porn. It should be noted that the representation of irrumatio and gagging are not exclusively reserved for girl-on-guy scenes; Tom Bolt Media's specialist gay line Gag the Fag, for example, is now on its sixth instalment, whilst Killergram's European Bitch Funkers VII incorporates depictions of choking and gagging into scenes between women.

Whilst gagging may be most visible within heterosexual porn cultures, then, it also has a presence within non-hetero adult entertainment, and this presence needs to be addressed. How do these kinds of movies problematize the idea of facial fluids as a stand in for the cum shot, or relate to theories about irrumatio's attempts to articulate a frenzy of the visible? Is this material simply an attempt to capitalize on the popularity of the hetero version of the subgenre, or does it have its own traditions and its own aesthetics? In what ways might gendered identifications be said to operate within non-heterosexual gagging porn, and how does the staging of gagging scenarios within lesbian pornography utilize and subvert ideas of the phallus? All of these questions suggest that this kind of porn should be explored in order to ascertain whether it can offer us a new perspective on irrumatio and gagging. As this article has demonstrated, gagging porn is far from the reductive and monolithic demonstration of violence against women that many have supposed it to be. Now we need to go further in our attempts to interrogate, problematize, and perhaps queer, a practice that seems at first glance like little more than a hyperbolic a demonstration of gendered aggression.

\section{Notes}

${ }^{1}$ Linda Williams uses this term as part of her comparison of pornography and the movie musical, during which she suggests that numbers are something like bodily spectacles which function "as events themselves within the larger structure" of the filmic text (130). 
${ }^{2}$ Compare this to "Cum-eating," with a relatively meagre 55 entries, or "Glory Holes" with 54 titles. Interestingly, "Cunnilingus," an act often prominently absent from general gonzo fare, does considerably better even than gagging, with a total of 3919 dedicated entries.

${ }^{3}$ It has a limited presence within avant garde cinema and art video installations - see "Hoist," Matthew Barney's contribution to the 2006 collection of art shorts Destricted, for example - but pornography is the chief vehicle for representing male ejaculate within contemporary visual culture.

${ }^{4}$ The exception being tears which, Kristeva suggests, do not have "any polluting value" (71).

${ }^{5}$ The role of the "creampie" - in which an internal ejaculation is made visible as it seeps out of the female bodily interior - is interesting here, and the way in which it speaks to a concern with the visualization of female sexual pleasure remains to be explored. I am indebted to Michael O'Rourke for bringing this idea to my attention.

${ }^{6}$ Indeed, the eighteenth-century thinker Adam Smith suggests that pain "is a more pungent sensation than pleasure" (53), and may therefore be easier to communicate and to discern. If "our sympathy with pain, though it falls greatly short of what is naturally felt by the sufferer, is generally a more lively and distinct perception than our sympathy with pleasure" (53), then suffering may be a more efficient and successful vehicle for a generalized frenzy of the visible.

${ }^{7}$ This quote is taken from an article in the New York Times about Peter Acworth, then head of the BDSM porn site Kink.com, whose company developed a list of self-imposed taboos and prohibitions against types of content.

${ }^{8}$ As Maddison notes, gonzo is "increasingly replacing the high-budget narrative features that dominated the hardcore market in the 1990s and early 2000s" (38).

${ }^{9}$ Krzywinska makes a similar point in relation to porn's attempt to visualize female ejaculations: "The relocation of ejaculation to the vagina, as opposed to the conventional penis, is to make it work as a specular index of pleasure and desire, but most importantly making the female orgasm visible" (40).

10 This statement is somewhat overbroad in its generalizations; some male porn stars, including Rocco Siffredi, can be very vocal in terms of linguistic and non-linguistic expressions of desire.

${ }^{11}$ Although, admittedly, the responses of female performers in gagging pornography are often muffled by the acts they are performing.

\section{Bibliography}

Boyle, Karen. "Epilogue: How Was it For You?" Everyday Pornography. Ed. Boyle. Abingdon: Routledge, 2010. pp. 201-211.

Brinkema, Eugenie. "Celluloid is Sticky: Sex, Death, Materiality, Metaphysics (in Some Films by Catherine Breillat)." Women: A Cultural Review 17:2 (2006): pp. 147-170. 
Dines, Gail. Pornland: How Porn has Hijacked Our Sexuality. Boston: Beacon Press, 2010.

Falk, Pasi. "The Representation of Presence: Outlining the Anti-aesthetics of Pornography." Theory, Culture \& Society 10:1 (1993): 1-42. Sage. Web. 20. Jan. 2011.

Herman, R.D.K. "Playing with Restraints: Space, Citizenship, and BDSM." Geographies of Sexualities: Theory, Practices and Politics. Ed. Kath Browne, Jason Lim, and Gavin Brown. Farnham: Ashgate, 2007. pp. 89-100.

. Jensen, Robert. Getting Off: Pornography and the End of Masculinity. Cambridge, MA: South End Press, 2007.

Kristeva, Julia. Powers of Horror: An Essay on Abjection. Trans. Leon S. Roudiez. New York: Columbia University Press, 1982.

Krzywinska, Tanya. "The Dynamics of Squirting: Female Ejaculation and Lactation in Hardcore Film." Unruly Pleasures: The Cult Film and its Critics. Ed. Xavier Mendik and Graeme Harper. Guildford: FAB Press, 2000. pp. 31-45.

Maddison, Stephen. "'Choke on it Bitch!': Porn Studies, Extreme Gonzo and the Mainstreaming of Hardcore." Mainstreaming Sex: The Sexualization of Western Culture. Ed. Feona Attwood. London: I. B. Tauris, 2009. pp. 37-53.

Mooallem, Jon. "A Disciplined Business." New York Times. 29 April 2007. Web. 22 March 2010. Moore, Lisa Jean, and Juliana Weissbein. "Cocktail Parties: Fetishizing Semen in Pornography Beyond Bukkake." Everyday Pornography. Ed. Karen Boyle. Abingdon: Routledge, 2010. pp. 77-89.

O'Toole, Laurence. Pornocopia: Porn, Sex, Technology and Desire. London: Serpent's Tail, 1999.

Paul, Pamela. Pornified: How Pornography is Damaging Our Lives, Our Relationships, and Our Families. New York: Holt, 2005.

Shamoon, Deborah. "Office Sluts and Rebel Flowers: The Pleasures of Japanese Pornographic Comics for Women." Porn Studies. Ed. Linda Williams. Durham, NC: Duke University Press, 2004. pp. 77-103.

Smith, Adam. The Theory of Moral Sentiments. Cambridge: Cambridge University Press, 2004.

Stüttgen, Tim. "Before Orgasm: Fifteen Fragments on a cartography of Post/Pornographic Politics". Post/Porn/Politics: Queer Feminist Perspectives on the Politics of Porn Performance and Sex Work as Cultural Production. Ed. Stüttgen. Berlin: b_books, 2009. pp. 10-21.

---. "Disidentification in the Center of Power: The Porn Performer and Director Belladonna as a Contrasexual Producer: A Letter to Beatriz Preciado." Post/Porn/Politics: Queer Feminist Perspectives on the Politics of Porn Performance and Sex Work as Cultural Production. Ed. Stüttgen. Berlin: b_books, 2009. pp. 41-55. 
Williams, Linda. Hard Core: Power, Pleasure, and the "Frenzy of the Visible." Berkeley: University of California Press, 1999.

\section{RECORDINGS / WEBSITES}

"Choke on My Cock." Hot Movies. VODconcepts.com, 2012. Web. 25 Oct. 2012. <http://www.hotmovies.com/video/217324/Choke-On-My-Cock/>.

"Fuck Her Throat." Hot Movies. VODconcepts.com, 2012. Web. 25 Oct. 2012. <http://www.hotmovies.com/video/148754/Fuck-Her-Throat-2/>.

Gag Factor. JM Productions, n.d. Web. 22 March 2010. <http://www.gagfactor.com/gagfactordotcom.html>.

Gag Factor 15. Dir. Jim Powers. JM Productions, 2004. Web. 08 Nov. 2009. <http://www.hotmovies.com/video/98641/Gag-Factor-15/?>.

"Hoist." Dir. Matthew Barney. Destricted. Revolver Entertainment, 2006. DVD.

"Adult Video Categories." Hot Movies. VODconcepts.com, 2012. Web. 25 Oct. 2012. <http://www.hotmovies.com/categories.php?>.

Rocco: Animal Trainer 8. Dir. Rocco Siffredi. Evil Angel, 2002. Web. 25 Oct. 2012.<http://www.hotmovies.com/video/133679/Rocco-Animal-Trainer-8/>.

"Sloppy Head 4." Hot Movies. VODconcepts.com, 2012. Web. 25 Oct. 2012. <http://www.hotmovies.com/video/209462/Sloppy-Head-4-Disc-2-/>.

Throat Gaggers. Red Light District, 2012. Web. 25 Oct. 2012. < http://www.throatgaggers.com/\#>

Throat Gaggers 13. Dir. Juan Cuba. Red Light District, 2007. Web. 25 Oct. 2012. <http://www.hotmovies.com/video/108894/Throat-Gaggers-13/>. 